\title{
BASIC CONSIDERATIONS IN THE CONSERVATIVE CARE OF HEAD INJURIES
}

\author{
A. R. HunTER, M.D., F.R.F.P.S.G., F.F.A.
}

Visiting Anaesthetist at the Royal Infirmary, Manchester, and Thoracic Unit, Baguley Hospital; Anaesthetist, Stepping Hill Hospital, Stockport; Clinical Tutor Anaesthetist, University of Manchester

The care of head injuries can be divided into two integral parts: the management of the neurological consequences of the injury to the brainthat is, the directly neurosurgical portion-and the conservative care of the patient during his period of unconsciousness. This latter consists essentially of the immediate resuscitation of the patient, the maintenance of the patency of his airway in the early stages of the illness, and subsequently the prevention or, if this is not possible, the prompt recognition and treatment of pulmonary infection on the one hand and the maintenance of the fluid balance and nutrition on the other. Two other important matters may also present themselves for attention, viz. the control of restlessness and the resuscitation of the apnoeic patient.

Many of these aspects of the care of head injuries came into prominence about the beginning of the present decade. ${ }^{1}$ They were discussed by Northfield, ${ }^{10}$ Lewin ${ }^{5,}{ }^{6}$ and others. More recently the Newcastle team ${ }^{7}, 8$ have presented their views. A rather more conservative outlook has been held by the author and his colleagues and this paper sets them forth.

The two problems which present themselves immediately on the admission of the patient are the maintenance of a clear airway and the replacement of blood loss.

\section{Care of the Airway}

The airway of a patient who is unconscious as a result of brain trauma is at risk for exactly the same reasons as that of an anaesthetized patient. The results of respiratory obstruction, however, even if it is only partial, are more disastrous than in almost any other form of unconsciousness, for the influences which can increase the bulk of a normal brain are even more active when the brain is damaged and already tending to become oedematous. A rise in blood $\mathrm{CO}_{2}$ content and the occurrence of abdominal respiratory straining are by far the most important of these factors.
Respiratory obstruction leads directly to carbon dioxide accumulation and indirectly to abdominal straining to overcome it. It is therefore a most potent cause of increase in the bulk of any brain and, in one already damaged by trauma, such an additional increase in intracranial tension may make the difference between the ultimate recovery and death of the patient.

Respiratory obstruction is prevented and treated in the comatose patient by exactly the same methods as those employed in everyday anaesthesia. Thus the patient is nursed on his side and if soft parts in the throat or trismus still cause obstruction an airway is inserted. The all-metal Waters instrument which cannot be bitten is better than the Guedel, which is mainly made of soft rubber.

A nasopharyngeal tube can be used where the nasal airway is adequate, but very often the nose will also have been injured and local swelling will cause compression and narrowing of any tube there. In addition, it is all too easy to abrade the nasal mucous membrane while passing such a tube. The resulting bleeding not only adds immediately to the obstruction of the airway, but the blood may be inhaled into the air passages with the expected adverse results. Preliminary spraying of the nose with an adrenaline solution with or without cocaine reduces, but does not abolish, the risk of producing epistaxis.

\section{Endotracheal Tubes}

An endotracheal tube will, of course, provide a clear and unobstructed airway. If possible, it is best passed through the nose, for it is then easier to fix and its patency, such as it is, is assured. On the other hand, concurrent nasal injury, or gross septal deflection, may so seriously reduce the lumen of a nasal tube, and add to the risk of epistaxis during its passage, that the end result may indeed be not relief, but aggravation of respiratory obstruction. An oral endotracheal tube is easy to pass, but difficult to fix. Though the 
patency of such a tube is usually more certain, there is always a danger that the jaws will close on it and obliterate its lumen. A London hospital prop offers some protection, but can all too readily fall out or be spat out. Either type of endotracheal intubation is best performed under topical anesthesia induced first by spraying down an airway and subsequently by spraying the larynx exposed by a Macintosh laryngoscope.

An endotracheal tube provides not only a clear airway, but is also a means of passing a suction catheter into the bronchi. It is, however, a total bar to swallowing and to some extent must prop the glottis open and thus predispose to the inhalation of saliva and other material in the pharynx. For this reason, and because of the risk of mechanical obstruction of the tube, this type of airway calls for the closest supervision of the patient by the nursing staff. It should be regarded as an interim measure to tide a patient over a period in which the prognosis is doubtful. It will most often be thus used in those over 60 years of age.

There is a limit to the length of time that an endotracheal tube may be left in situ. In the presence of gross tracheo-bronchial infection the cords and tracheal mucous membrane are liable to show signs of commencing ulceration in 48 hours. On the other hand, endotracheal tubes have in the past been left for a week without ill effects in patients suffering from respiratory obstruction without infection. This procedure is not, however, to be recommended today in view of the enlightened current outlook on tracheostomy.

Tracheostomy will, of course, provide a clear airway in any patient, but where recovery of consciousness is going to occur in a day or two it should not be necessary. Equally where total ultimate recovery is unlikely this operation, if performed too early, may lead to the perpetuation of the life of one so handicapped mentally that he is a burden to himself and to others. In general, a tracheostomy is regarded more as a port for the removal of secretions and foreign material from the airway than as a means of ensuring its patency.

\section{Treatment of Shock}

The next problem is the treatment of shock. Usually this is not in evidence in the simple closed head injury. Indeed, it is a fair presumption that where the blood pressure is low some other structures in the body have also been damaged, though a fair amount of blood loss can occur from a large scalp laceration. When hypotension is present as a result of other complicating injuries the risk of producing further intracranial bleeding by raising the blood pressure is always difficult to assess. In general, however, it is probably safe to give a transfusion sufficient to raise the blood pressure to $100 \mathrm{~mm}$. Hg., irrespective of its previous level. Prolonged hypotension will almost certainly reduce the chances of survival of a devitalized brain.

\section{Aspirated Foreign Material}

When the airway has been cleared and shock has been treated, the next stage in the resuscitation is the clearance of any foreign material which has entered the tracheo-bronchial tree during the early period of unconsciousness. This foreign material may be vomitus, blood from a concomitant nasal injury or from fracture of the skull base or even cerebrospinal fluid. In the first few hours of unconsciousness there is, of course, the risk of further inhalation and for this reason also the semi-prone position should be used for nursing these patients. Wherever it is permissible for surgical reasons a slight head down slope is also desirable. Under no circumstances should the patient be allowed to lie in the dorsal position. The trachea is normally inclined at $30^{\circ}$ to the coronal plane and no bed elevator will reverse this inclination with the patient on his back and thus prevent the downward drainage of foreign material from the back of the pharynx.

Posture alone is of some value in removing foreign material from the lungs in such cases, but often a formal tracheo-bronchial toilet is necessary. Any standard method will serve. Either a suction catheter may be passed down a shielding endotracheal tube or a formal bronchoscopy may be employed, the choice being governed by the skill of the available operator. The urgency of this procedure depends on the amount of soiling which seems to have occurred. Where, however, it is possible to do so, there is something to be said for delaying tracheo-bronchial toilet and the inevitable accompanying spasm of coughing until some time has been allowed for the damaged intracranial veins to be closed off by blood clot, as they will be some 12 to 24 hours after the injury.

\section{Resuscitation of the Apnoeic Patient}

Probably the most difficult problem of all in the management of the patient with a severe head injury is that of the appropriate treatment of respiratory failure. Where the intracranial pressure has risen so high that apnoea has occurred the circulation can always be kept going for many hours thereafter by artificial respiration. There must, therefore, be a temptation to embark on this every time. In fact, however, cerebral compression sufficient to cause apnoea always means that irreparable damage will be done to the brain within a very short period, and after this artificial respiration merely postpones the inevitable. For 
this reason it is necessary to institute artificial respiration wenever breathing stops, because of a recognizable localized and promptly treatable compression of the brain, as, for example, an extradural haematoma or when apnoea follows an anoxic episode produced by acute respiratory obstruction. On the other hand, where there is no prospect of immediate surgical relief of the pressure causing the apnoea, it is unwise to embark on what ultimately must prove to be the most fruitless of all restorative measures.

\section{Subsequent Care of Prolonged Unconsciousness}

Once the dangers of the immediate posttraumatic period have been passed there arise the problems of the maintenance of the vital functions in a patient unconscious for a long period. These consist essentially of routine nursing care, of feeding and of the prevention of infection of the lungs.

The lungs are liable to be soiled as a result of the inspiration of pharyngeal contents, which may be blood, saliva, vomitus or more rarely cerebrospinal fluid. Also, if the patient is nursed solely in one position, as he may require to be if he has other injuries, secretion will certainly accumulate in the bronchi of the dependent lobes and atelectasis and subsequent infection will occur. Finally, no matter how carefully the catheter for tracheal aspiration is cleaned, or even if a fresh tube is used every time one is requirc d, the resident organisms in the ward will almost certainly find their way into the patient's bronchi, though they may be prevented from going further by the methods used to promote tracheo-bronchial drainage.

To ensure that serious pulmonary infection does not occur the following attention is required. First, the patient must be properly nursed in an adequately staffed unit where it will be possible to ensure his regular change in position, the treatment of his pressure areas, the aspiration of his respiratory tract whenever moist sounds indicate that this is necessary, and the changing of his bed immediately it becomes soiled. Secondly, adequate physiotherapy must be available, particularly in patients tending to develop sputum retention, twice a day on all seven days of the week. Thirdly, the patient's chest must be examined every day by someone competent to recognize the signs of sputum retention and incipient atelectasis. This physical examination affords a valuable check on the effectiveness of postural drainage and physiotherapy, and very often it will be found that an area of lung which was not aerating one day can be brought back into action simply by making an appropriate modification in the patient's posture and intensifying the physiotherapy directed to the draining of the appropriate bronchus.

Posture and physiotherapy alone will not prevent broncho-pulmonary infection. Prophylactic antiinfective agents are also required. Penicillin, I mega unit twice daily, is the obvious choice, but in view of the mixed flora invariably found after bronchial soiling it is wise to give sulphamethazine ( 0.5 g. six-hourly) also as soon as the urine output is satisfactory. A very real problem arises when signs of consolidation appear in a patient already on these drugs. For it is obvious that the organism is sensitive to neither and the therapeutic choice now lies between chloramphenicol, which may interfere with blood formation, and the tetracyclines, whose administration is liable to be complicated by enteric infection with a resistant staphylococcus. This is doubly likely, on the one hand, because the patient is in hospital, where such organisms are indigenous, and, on the other, because the milky diet used in such cases completely inhibits the normally fairly effective antiseptic action of the gastric hydrochloric acid. 'To some extent this difficulty can be overcome by the use of intravenous tetracyclines, and this is probably the method of choice.

\section{Tracheostomy}

Finally, there remains the question of tracheostomy as a means of preventing pulmonary infection. Much has been written about this recently and there are very real dangers in all this publicity. For it may lead to a belief that the most important factor in ensuring the survival of a patient with a bad head injury is the performance of this operation, with the subsequent neglect of all the other items of detailed medical and nursing care which are so necessary in this type of case. Secondly, it may lead to the carrying out of the operation in cases where it is not strictly necessary or even where it is frankly undesirable. As the complications of tracheostomy are numerous and by no means all preventable, the result in some cases may be to reduce rather than to improve the patient's chances of survival.

The essential indication for tracheostomy is the inability to ensure by other means the patency and adequate drainage of the patient's tracheo-bronchial tract during the period of unconsciousness. The reason for this may simply be lack of adequate or sufficiently skilled nursing or physiotherapist staff. The presence of other injuries which necessitate the immobilization of the patient in one position will almost certainly make tracheostomy necessary. So also will injuries of the thorax which limit coughing and fix the chest wall. Lastly, the immediate effect of the depression of the central nervous system which accompanies a serious head 
injury is a suppression of the cough reflex. In a patient with a pre-existing bronchitis a flare up of the infection inevitably ensues. This sets up two vicious cycles. First, sputum accumulation occurs and aggravates the infection. Secondly, the accompanying interference with oxygen uptake deepens the unconsciousness and further reduces the activity of the cough reflex with more sputum retention. Only frequent aspiration of the respiratory passages will break these vicious cycles, and for this tracheostomy is very frequently necessary.

There is, however, a price to be paid in complications for tracheostomy. First, the tube may abrade the posterior wall of the trachea and cause ulceration. This ulcer will most certainly become infected and the exudate from it will add to the amount of secretion which must be aspirated from the respiratory tract. Secondary haemorrhage from such ulcers is by no means unknown. It is rarely fatal, but it is not difficult to conceive what might be the result of the loss of a fair amount of blood into the airway of an inadequately supervised patient.

Secondly, tracheostomy deprives the lower respiratory tract of its normal humidifying mechanism for inspired air. In a minority of patients the ciliated epithelium of the respiratory tract cannot accommodate itself to this and pours out a fibrinous exudate. This may appear in quantity sufficient to obstruct the airway. Fortunately, this complication is amenable to treatment with a steam tent and will be prevented entirely if all patients who have this operation are given artificially humidified air to breathe for the first 48 hours. If simultaneous hypothermia is necessary, a nebulizer may be used to provide the necessary humid atmosphere rather than a steam tent.

Lastly, in inadequately supervised patients tracheostomies can become obstructed, sometimes by clots of fibrin, but more often because the tube has slipped out of the trachea into the tissues of the neck. As the patient strains to overcome the resulting blockage of the airway, the pressure in the tissues of the neck becomes negative and air is drawn into them. The first result is local surgical emphysema. Then, as the negative pressure draws the air yet further down, mediastinal emphysema occurs. Finally, as the pleura gives way under the strain of the enormous negative pressure generated in it by the forced inspiration a pneumothorax is formed. ${ }^{2}$ Pus can be carried down with the air with development of mediastinitis and even pyopneumothorax. The writer has no personal experience of such accidents, but they have been mentioned to him by other workers.

It will, therefore, be apparent that, though tracheostomy is of very real value in suitable cases, it is not a procedure to be undertaken lightly, nor in the absence of a definite and positive indication for it.

\section{Feeding}

The second part of the care of head injuries consists of the maintenance of the fluid balance and the subsequent provision of a suitable dietary intake in a patient who is unable to swallow.

The unconscious patient, whatever the cause of his coma, be it a head injury or some disease process, has a profound depression of his swallowing reflexes. When fluid is placed in his mouth at best only a portion of it is swallowed, while that which is rejected is very liable to find its way down through the larynx and trachea into the lungs, there to give rise to the usual consequences of the inhalation of foreign material. For this reason it is essential that comatose patients should be fed by means of a stomach tube.

In those lightly unconscious what remains of the swallow reflex may be just sufficient to allow the tube to be passed in the normal way or, in an edentulous patient, with the help of a guiding finger in the back of the throat. Alternatively, in those more comatose the tube may be picked up with a Magill's forceps after it has been passed through the nose into the back of the throat and, if it is sufficiently rigid, be guided down the oesophagus into the stomach. If it is felt undesirable to use a semi-rigid tube, the most pliable of Ryle's tubes can be passed by the oral route down a shielding endotracheal tube which has previously been passed under vision into the oesophagus. This technique calls for the most generous lubrication of the Ryle's tube to ensure that it slides freely. Also, as the cardiac sphincter normally opens some six seconds after the entry of a bolus into the upper oesophagus, it is desirable that a Ryle's tube passed in this way should be pushed down the inserted endotracheal tube without delay.

It is difficult to fix a Ryle's tube passed through the mouth. For this reason its upper end should, after the removal of the sheathing endotracheal tube, be drawn in a retrograde direction into the nose. This is achieved by passing a fine rubber catheter through the nose into the back of the throat. Its end is then brought out through the mouth and to it the Ryle's tube is attached. The catheter is then withdrawn until it comes out through the nostril, bringing the Ryle's tube with it.

\section{Fluid Balance}

The maintenance of the fluid balance is a serious problem in the patient with a head injury. In the vast majority of cases the patient will be allowed to lie for some 24 hours in the hope that spon- 
taneous recovery will occur before any attempt is made to have him drink. During this period he will have lost a considerable proportion of his body fluid. In cases with other complicating injuries there will have been, in addition, a considerable deviation of the plasma volume as fluid lost into the tissues at the site of injury. The presence of bruising of the bowel, too, will also tend to produce gastrointestinal stasis, with accompanying disturbance of the electrolyte balance. Lastly, there are a number of electrolyte disturbances which are said to be the specific result of injury of the brain. ${ }^{3,} 9$ Even true renal failure may occur. ${ }^{11}$

The first problem is the administration of water. This is best done via the Ryle's tube in the form of a $5 \%$ dextrose solution. It is, on the one hand, vital that the patient should receive as much fluid as possible, yet, on the other hand, it is essential that fluid should not be continuously pushed into a stomach which is not passing it on. For if this is done it is more than possible that a torrential regurgitation. with inhalation. of the vomitus and asphyxia will result. Two ounces of $5 \%$ dextrose should therefore be injected down the Ryle's tube every two hours for the first 12 hours after it has been passed. Thereafter the fluid intake should be increased by steps of $2 \mathrm{oz}$. until the patient is receiving $96 \mathrm{oz}$. in 24 hours; that is, 8 oz. two-hourly. By the same token an empty syringe must be attached to the stomach tube and the stomach aspired to dryness immediately before fluid is injected down the tube, and only if there is a negligible residue is it safe to increase the amount of fluid. given. Five per cent. dextrose in water should not be employed as a tube feed for more than 48 hours; if it is given for a long period, carbohydrate fermentation diarrhoea will almost certainly result.

Intravenous fluid administration will be necessary where there has been a prolonged period of fluid deprivation or where gastro-intestinal bruising has led to stasis, with the result that it is impossible to give the necessary amount of fluid by Ryle's tube. It will, however, often be a matter of difficulty to keep a needle or cannula in the vein of a patient who is being moved from side to side hourly. Whatever method of fluid administration is employed, it is vital that the intake should be carefully recorded. In addition, the serum electrolytes should be frequently determined. Suitable modifications in the amount of fluid to be given will then be indicated by changes in the serum sodium.

\section{Diet}

After the first 24 hours it is obviously desirable to provide the patient with more than water and energy in the form of a dextrose solution. Unconscious patients are liable to wasting and a diet containing $120 \mathrm{~g}$. of protein in 24 hours is the ultimate aim. This is best provided by the addition of increasing amounts of eggs and boiled milk to the tube feed. The proprietary food, 'Complan', although sometimes valuable, seems too liable to provoke diarrhoea for routine administration. Wasting can be reduced by administration of an anabolic steroid such as norethandrolone which increases protein synthesis.

However desirable a high protein intake is from the point of view of the prevention of wasting, it cannot always be employed from the start. The blood urea rises rapidly after a head injury and it is generally believed that it is unwise to attempt to press the administration of protein until the blood urea is less than $80 \mathrm{mg}$. $/ 100 \mathrm{ml}$.

Because of restlessness or involuntary movement, intravenous feeding is difficult in such patients. The need for the administration of food, as opposed to fluid, by the intravenous route should, however, be one which arises only very rarely.

\section{Care of the Eyes}

Comatose patients often lie with their eyes partly open. Tear secretion is depressed partly as a result of the coma itself, but usually more so because of the accompanying dehydration. Blinking does not occur. As a result it is very common for the corneae to become dry, to exfoliate and ulcerate. For this reason the eyes of unconscious patients should receive special attention from the nursing staff, be irrigated if they are unduly dry and have appropriate anti-infective solutions, such as $10 \%$ sulphacetamide, dropped into them at regular intervals should they become reddened. Every possible precaution, including, if need be, the covering of the eyes, should be taken to avoid accidental trauma to the cornea.

\section{Sedation}

Few patients cause more difficulty in a general surgical ward than those restless after head injury. In the early stages of the condition the patient's level of unconsciousness is such a vital guide to his progress that no form of sedation is permissible. It is, however, always worth while looking at the lower abdomen of a restless patient to see whether a distended bladder is the cause of the trouble, for its drainage will very often abolish restlessness.

Later, in the natural history of a head injury, there is often a period of restlessness during the stage of recovery. If this occurs some days after the injury and follows a period of progressive improvement it is then safe to control the restlessness with chlorpromazine. As much as $5^{\circ} \mathrm{mg}$. 
four-hourly may be given. It must be emphasized again, however, that this form of sedation is applicable only where the surgeon no longer requires the patient's state of unconsciousness as a guide to his progress. It is, above all, vital that chlorpromazine sedation should not be employed to control restlessness in a patient who has become more drowsy than he was previously.

There are many other aspects to the conservative care of head injuries and of these the most notable is that of the appropriate use of hypothermia. This has, however, been dealt with elsewhere by a number of authors. Further, only a minority of cases require this form of treatment. It is, therefore, not a basic consideration like the other matters referred to in this article. Further information on it should be sought in the papers of Maciver and others 7 , and of Matheson ${ }^{8}$.

In general, the conservative care of head injuries is essentially a matter of team work, in which the surgeon and the anaesthetist, the radiologist and the biochemist all have a vital role to play. It is, too, perhaps that branch of medicine above all others where success is impossible with- out the devoted and conscientious care of the patient by the nursing staff and the physiotherapists. It is only where such total team work is possible that the best results will be obtained in this most fascinating yet difficult work.

\section{Acknowledgments}

My thanks are due to Professor Sir Geoffrey Jefferson, who first introduced me to this work, and to Mr. R. T. Johnson and Mr. J. M. Potter, who continue to encourage me in it.

\section{REFERENCES}

I. BRYCE-SMITH, R. (1950), Brit. med. F., ii, 322.

2. DARK, J. F. (1952), Lancet, i, 398.

3. HIGGINS, G., LEWIN, W., O'BRIEN, J. R. P., and TAYLOR, W. H. (195 I), Ibid., i, I 295.

4. LASSMAN, L. P., THOMSON, C. W., and McLEOD, I: (1958), Ibid., ii, 544 .

5. LEWIN, W. (1953), Brit. med. f., i, 1239.

6. LEWIN, W. (1954), Proc. Roy. Soc. Med., 47, 865.

7. MACIVER, I. N., FREW, I. J. C., and MATHESON, J. G. (1958), Lancet, i, 391.

8. MATHESON, J. G., THOMSON, C. W., and WHITBY, J. D. (1959), Anaesthesia, 14, 168.

9. METZ, R. J. S., and COOPER, W. (1958), Brit. med. F., i, 435.

10. NORTHFIELD, D. C. (1953), Ibid., i, 90.

I . TAYLOR, W. H. (1957), Lancet, ii, 703.

\section{DIA B ETES}

(Postgraduate Medical fournal, May 1959)

Price 6s. 6d. post free

ORAL HYPOGLYCAEMIC DRUGS

J. D. H. Slater, M.A., M.B., M.R.C.P.

DIABETES IN CHILDREN

James Robertson, M.B., M.R.C.P.

AETIOLOGICAL FACTORS IN DIABETES

D. A. Pyke, M.D., M.R.C.P.

PLASMA INSULIN ACTIVITY IN DIABETICS

J. Vallance-Owen, M.A., M.D. (Cantab.), M.R.C.P.

\section{AUTONOMIC NEUROPATHY IN DIABETES MELLITUS \\ Harry Keen, M.B., M.R.C.P.}

SOCIAL PROBLEMS OF THE DIABETIC

Iris Holland Rogers

PREDIABETES-A SYNTHESIS

W. P. U. Jackson, M.A., M.D., M.R.C.P., D.C.H.

THE OCULAR COMPLICATIONS OF DIABETES

P. D. Trevor-Roper, M.A., M.B., B.Chir., F.R.C.S.

THE DETECTION OF IAATENT DIABETES Joan B. Walker, M.D.

THE GLUCOSE TOLERANCE TEST

Joyce D. Baird, M.A., M.B.Ch.B., and Leslie J. P. Duncan, B.Sc., M.B.Ch.B., M.R.C.P.E. 